\title{
On Functional Inequalities Originating from Module Jordan Left Derivations
}

\author{
Hark-Mahn Kim, ${ }^{1}$ Sheon-Young Kang, ${ }^{2}$ and Ick-Soon Chang ${ }^{3}$ \\ ${ }^{1}$ Department of Mathematics, Chungnam National University, Daejeon 305-764, South Korea \\ ${ }^{2}$ Department of Industrial Mathematics, National Institute for Mathematical Sciences, \\ Daejeon 305-340, South Korea \\ ${ }^{3}$ Department of Mathematics, Mokwon University, Daejeon 302-729, South Korea
}

Correspondence should be addressed to Ick-Soon Chang, ischang@mokwon.ac.kr

Received 18 February 2008; Revised 1 May 2008; Accepted 19 May 2008

Recommended by András Rontó

We first examine the generalized Hyers-Ulam stability of functional inequality associated with module Jordan left derivation (resp., module Jordan derivation). Secondly, we study the functional inequality with linear Jordan left derivation (resp., linear Jordan derivation) mapping into the Jacobson radical.

Copyright () 2008 Hark-Mahn Kim et al. This is an open access article distributed under the Creative Commons Attribution License, which permits unrestricted use, distribution, and reproduction in any medium, provided the original work is properly cited.

\section{Introduction and Preliminaries}

Let $\mathcal{A}$ be an algebra over the real or complex field $\mathbb{F}$ and let $\mathcal{M}$ a left $\mathcal{A}$-module (resp., $\mathcal{A}$ bimodule). An additive mapping $d: \mathcal{A} \rightarrow \mathcal{M}$ is said to be a module left derivation (resp., module derivation) if $d(x y)=x d(y)+y d(x)$ (resp., $d(x y)=x d(y)+d(x) y)$ holds for all $x, y \in \mathcal{A}$. An additive mapping $d: \mathscr{A} \rightarrow \mathcal{M}$ is called a module Jordan left derivation (resp., module Jordan derivation) if $d\left(x^{2}\right)=2 x d(x)$ (resp., $\left.d\left(x^{2}\right)=x d(x)+d(x) x\right)$ is fulfilled for all $x \in \mathcal{A}$. Since $\mathcal{A}$ is a left $\mathcal{A}$-module (resp., $\mathcal{A}$-bimodule) with the product giving the module multiplication (resp., two module multiplications), the module left derivation (resp., module derivation) $d$ : $\mathscr{A} \rightarrow \mathcal{A}$ is a ring left derivation (resp., ring derivation) and the module Jordan left derivation (resp., module Jordan derivation) $d: \mathcal{A} \rightarrow \mathcal{A}$ is a ring Jordan left derivation (resp., ring Jordan derivation). Furthermore, if the identity $d(\lambda x)=\lambda d(x)$ is valid for all $\lambda \in \mathbb{F}$ and all $x \in \mathcal{A}$, then $d$ is a linear left derivation (resp., linear derivation, linear Jordan left derivation, and linear Jordan derivation).

It is of interest to consider the concept of stability for a functional equation arising when we replace the functional equation by an inequality which acts as a perturbation of the 
equation. The study of stability problems had been formulated by Ulam [1] during a talk in 1940: Under what condition does there exist a homomorphism near an approximate homomorphism? In the following year, Hyers [2] was answered affirmatively the question of Ulam for Banach spaces, which states that if $\varepsilon>0$ and $f: x \rightarrow y$ is a map with $x$ a normed space, $y$ a Banach space such that

$$
\|f(x+y)-f(x)-f(y)\| \leq \varepsilon
$$

for all $x, y \in \mathcal{X}$, then there exists a unique additive map $T: x \rightarrow y$ such that

$$
\|f(x)-T(x)\| \leq \varepsilon
$$

for all $x \in \mathcal{X}$. A generalized version of the theorem of Hyers for approximately additive mappings was given by Aoki [3] in 1950 (cf. also [4]) and for approximately linear mappings it was presented by Rassias [5] in 1978 by considering the case when inequality (1.1) is unbounded. Due to that fact, the additive functional equation $f(x+y)=f(x)+f(y)$ is said to have the generalized Hyers-Ulam stability property. The stability result concerning derivations between operator algebras was first obtained by Šemrl [6]. Recently, Badora [7] gave a generalization of the Bourgin's result [8]. He also dealt with the Hyers-Ulam stability and the Bourgin-type superstability of ring derivations in [9].

In 1955, Singer and Wermer [10] obtained a fundamental result which started investigation into the ranges of linear derivations on Banach algebras. The result, which is called the Singer-Wermer theorem, states that every continuous linear derivation on a commutative Banach algebra maps into the Jacobson radical. They also made a very insightful conjecture, namely, that the assumption of continuity is unnecessary. This was known as the Singer-Wermer conjecture and was proved in 1988 by Thomas [11]. The Singer-Wermer conjecture implies that every (or equivalently, linear left derivation) linear derivation on a commutative semisimple Banach algebra is identically zero which is the result of Johnson [12]. After then, Hatori and Wada [13] showed that a zero operator is the only ring derivation on a commutative semisimple Banach algebra with the maximal ideal space without isolated points. Note that this differs from the above result of Johnson. Based on these facts and a private communication with Watanabe [14], Miura et al. proved the the generalized HyersUlam stability and Bourgin-type superstability of ring derivations on Banach algebras in [14].

On the other hand, Gilanyi [15] and Rätz [16] proved that if $f$ is a mapping such that the functional inequality

$$
\|2 f(x)+2 f(y)-f(x-y)\| \leq\|f(x+y)\|,
$$

then $f$ satisfies the Jordan-von Neumann functional equation

$$
2 f(x)+2 f(y)=f(x+y)+f(x-y) .
$$

Moreover, Fechner [17] and Gilányi [18] showed the generalized Hyers-Ulam stability of the functional inequality (1.3).

The main purpose of the present paper is to offer the generalized Hyers-Ulam stability of functional inequality associated with module Jordan left derivation (resp., module Jordan left derivation). In addition, we investigate the functional inequality with linear Jordan left derivation (resp., linear Jordan derivation) mapping into the Jacobson radical. 


\section{Functional inequalities for module Jordan left derivations}

Throughout this paper, we assume that $k$ is a fixed positive integer.

Theorem 2.1. Let $\mathbb{A}$ be a normed algebra and let $\mathcal{M}$ be Banach left $\mathcal{A}$-module. Suppose that $f: \mathcal{A} \rightarrow \mathcal{M}$ is a mapping such that

$$
\|f(u)+f(v)+f(w)-2 x f(x)\| \leq\left\|\frac{1}{k} f\left(k u+k v+k w-k x^{2}\right)\right\|
$$

for all $u, v, w, x \in \mathcal{A}$. Then $f$ is a module Jordan left derivation.

Proof. Setting $x=0$ in (2.1) and using the Park's result [19], we see that $f$ is additive. Letting $u=v=0$ and $w=x^{2}$ in (2.1) gives

$$
\left\|f\left(x^{2}\right)-2 x f(x)\right\| \leq\left\|\frac{1}{k} f(0)\right\|=0
$$

for all $x \in \mathcal{A}$, which implies that $f\left(x^{2}\right)=2 x f(x)$ for all $x \in \mathcal{A}$. So we conclude that $f$ is a module Jordan left derivation. This completes the proof of the theorem.

We now establish the generalized Hyers-Ulam stability of functional inequality associated with module Jordan left derivation.

Theorem 2.2. Let $\mathcal{A}$ be a normed algebra and let $\mathcal{M}$ be a Banach left $\mathcal{A}$-module. Suppose that $f$ : $\mathcal{A} \rightarrow \mathcal{M}$ is a mapping for which there exists a function $\Phi: \mathscr{A}^{4} \rightarrow[0, \infty)$ such that

$$
\begin{gathered}
\sum_{j=0}^{\infty} 4^{j} \Phi\left(\frac{u}{2^{j+1}}, \frac{v}{2^{j+1}}, \frac{w}{2^{j+1}}, \frac{x}{2^{j+1}}\right)<\infty, \\
\|f(u)+f(v)+f(w)-2 x f(x)\| \leq\left\|\frac{1}{k} f\left(k u+k v+k w-k x^{2}\right)\right\|+\Phi(u, v, w, x)
\end{gathered}
$$

for all $u, v, w, x \in \mathcal{A}$. Then there exists a unique module Jordan left derivation $d: \mathcal{A} \rightarrow \boldsymbol{M}$ satisfying

$$
\|f(x)-d(x)\| \leq \sum_{j=0}^{\infty}\left[2^{j} \Phi\left(\frac{-x}{2^{j+1}}, \frac{-x}{2^{j+1}}, \frac{x}{2^{j}}, 0\right)+2^{j+1} \Phi\left(\frac{x}{2^{j+1}}, \frac{-x}{2^{j+1}}, 0,0\right)\right]
$$

for all $x \in \mathcal{A}$.

Proof. Letting $u=v=w=x=0$ in (2.3), we get

$$
\frac{3 k-1}{k}\|f(0)\| \leq \Phi(0,0,0,0)
$$

Since $\lim _{n \rightarrow \infty} 4^{n} \Phi(0,0,0,0)=0$, we have $\Phi(0,0,0,0)=0$. Hence $f(0)=0$. Let us take $u=v=$ $x, w=-2 x$ and $x=0$ in (2.3). Then we obtain

$$
\|2 f(x)+f(-2 x)\| \leq \Phi(x, x,-2 x, 0)
$$


for all $x \in \mathcal{A}$. Replacing $x$ by $-x / 2$ in the previous part, we get

$$
\left\|f(x)+2 f\left(\frac{-x}{2}\right)\right\| \leq \Phi\left(\frac{-x}{2}, \frac{-x}{2}, x, 0\right)
$$

for all $x \in \mathcal{A}$. Letting $u=x, v=-x$ and $w=x=0$ in (2.3), we arrive at

$$
\|f(x)+f(-x)\| \leq \Phi(x,-x, 0,0)
$$

for all $x \in \mathcal{A}$. Therefore by (2.7) and (2.8), we have

$$
\begin{aligned}
\left\|2^{l} f\left(\frac{x}{2^{l}}\right)-2^{m} f\left(\frac{x}{2^{m}}\right)\right\| & \leq \sum_{j=l}^{m-1}\left\|2^{j} f\left(\frac{x}{2^{j}}\right)-2^{j+1} f\left(\frac{x}{2^{j+1}}\right)\right\| \\
& \leq \sum_{j=l}^{m-1}\left[\left\|2^{j} f\left(\frac{x}{2^{j}}\right)+2^{j+1} f\left(\frac{-x}{2^{j+1}}\right)\right\|+\left\|2^{j+1} f\left(\frac{-x}{2^{j+1}}\right)+2^{j+1} f\left(\frac{x}{2^{j+1}}\right)\right\|\right] \\
& \leq \sum_{j=l}^{m-1}\left[2^{j} \Phi\left(\frac{-x}{2^{j+1}}, \frac{-x}{2^{j+1}}, \frac{x}{2^{j}}, 0\right)+2^{j+1} \Phi\left(\frac{x}{2^{j+1}}, \frac{-x}{2^{j+1}}, 0,0\right)\right]
\end{aligned}
$$

for all integers $l, m$ with $m>l \geq 0$ and all $x \in \mathcal{A}$. It follows that for each $x \in \mathcal{A}$ the sequence $\left\{2^{n} f\left(x / 2^{n}\right)\right\}$ is Cauchy and so it is convergent, since $\mathcal{M}$ is complete. Let $d: \mathcal{A} \rightarrow \mathcal{M}$ be a mapping defined by $(x \in \mathcal{A})$,

$$
d(x):=\lim _{n \rightarrow \infty} 2^{n} f\left(\frac{x}{2^{n}}\right)
$$

By letting $l=0$ and passing $m \rightarrow \infty$, we get inequality (2.4).

First of all, we note from (2.8) that

$$
\|d(x)+d(-x)\| \leq \lim _{n \rightarrow \infty} 2^{n}\left\|f\left(\frac{x}{2^{n}}\right)+f\left(\frac{-x}{2^{n}}\right)\right\| \leq \lim _{n \rightarrow \infty} 2^{n} \Phi\left(\frac{x}{2^{n}}, \frac{-x}{2^{n}}, 0,0\right)=0
$$

for all $x \in \mathcal{A}$. So we have $d(-x)=-d(x)$ for all $x \in \mathcal{A}$. Letting $u=x, v=y, w=-x-y$ and $x=0$ in (2.3), we find that

$$
\|f(x)+f(y)+f(-x-y)\| \leq \Phi(x, y,-x-y, 0)
$$

for all $x, y \in \mathcal{A}$. We obtain by (2.12) that

$$
\begin{aligned}
\|d(x)+d(y)-d(x+y)\| & =\|d(x)+d(y)+d(-x-y)\| \\
& =\lim _{n \rightarrow \infty} 2^{n}\left\|f\left(\frac{x}{2^{n}}\right)+f\left(\frac{y}{2^{n}}\right)+f\left(\frac{-x-y}{2^{n}}\right)\right\| \\
& \leq \lim _{n \rightarrow \infty} 2^{n} \Phi\left(\frac{x}{2^{n}}, \frac{y}{2^{n}}, \frac{-x-y}{2^{n}}, 0\right)=0
\end{aligned}
$$


for all $x, y \in \mathcal{A}$, that is, $d$ is additive. Setting $u=v=0$ and $w=x^{2}$ in (2.3) yields

$$
\left\|f\left(x^{2}\right)-2 x f(x)\right\| \leq \Phi\left(0,0, x^{2}, x\right)
$$

for all $x \in \mathcal{A}$. Using inequality (2.14), we get

$$
\left\|d\left(x^{2}\right)-2 x d(x)\right\|=\lim _{n \rightarrow \infty}\left\|4^{n} f\left(\frac{x^{2}}{4^{n}}\right)-2 x \cdot 2^{n} f\left(\frac{x}{2^{n}}\right)\right\| \leq \lim _{n \rightarrow \infty} 4^{n} \Phi\left(0,0, \frac{x^{2}}{4^{n}}, \frac{x}{2^{n}}\right)=0
$$

for all $x \in \mathcal{A}$, which means that $d\left(x^{2}\right)=2 x d(x)$ for all $x \in \mathcal{A}$. Therefore, we conclude that $d$ is a module Jordan left derivation.

Suppose that there exists another module Jordan left derivation $D: \mathcal{A} \rightarrow \mathcal{M}$ satisfying inequality (2.4). Since $D(x)=2^{n} D\left(x / 2^{n}\right)$ and $d(x)=2^{n} d\left(x / 2^{n}\right)$, we see that

$$
\begin{aligned}
\|D(x)-d(x)\| & =2^{n}\left\|D\left(\frac{x}{2^{n}}\right)-d\left(\frac{x}{2^{n}}\right)\right\| \\
& \leq 2^{n}\left[\left\|D\left(\frac{x}{2^{n}}\right)-f\left(\frac{x}{2^{n}}\right)\right\|+\left\|f\left(\frac{x}{2^{n}}\right)-d\left(\frac{x}{2^{n}}\right)\right\|\right] \\
& \leq 2 \sum_{j=n}^{\infty}\left[2^{j} \Phi\left(\frac{-x}{2^{j+1}}, \frac{-x}{2^{j+1}}, \frac{x}{2^{j}}, 0\right)+2^{j+1} \Phi\left(\frac{x}{2^{j+1}}, \frac{-x}{2^{j+1}}, 0,0\right)\right],
\end{aligned}
$$

which tends to zero as $n \rightarrow \infty$ for all $x \in \mathcal{A}$. So that $D=d$ as claimed and the proof of the theorem is complete.

Theorem 2.3. Let $\mathcal{A}$ be a normed algebra and let $\mathcal{M}$ be a Banach left $\mathcal{A}$-module. Suppose that $f$ : $\mathcal{A} \rightarrow \mathcal{M}$ is a mapping for which there exists a function $\Phi: \mathcal{A}^{4} \rightarrow[0, \infty)$ such that

$$
\sum_{j=0}^{\infty} \frac{1}{2^{j}} \Phi\left(2^{j} u, 2^{j} v, 2^{j} w, 2^{j} x\right)<\infty
$$

and inequality (2.3) for all $u, v, w, x \in \mathcal{A}$. Then there exists a unique module Jordan left derivation $d: \mathcal{A} \rightarrow \mathcal{M}$ satisfying

$$
\|f(x)-d(x)\| \leq \sum_{j=0}^{\infty} \frac{1}{2^{j+1}}\left[\Phi\left(2^{j} x, 2^{j} x,-2^{j+1} x, 0\right)+\Phi\left(2^{j+1} x,-2^{j+1} x, 0,0\right)+\frac{k+2}{3 k-1} \Phi(0,0,0,0)\right]
$$

for all $x \in \mathcal{A}$.

Proof. By the same reasoning as in the proof of Theorem 2.2, we find that

$$
\frac{1}{k}\|f(0)\| \leq \frac{1}{3 k-1} \Phi(0,0,0,0) .
$$

If we take $u=v=x, w=-2 x$ and $x=0$ in (2.3), then we get

$$
\|2 f(x)+f(-2 x)\| \leq \Phi(x, x,-2 x, 0)+\frac{1}{3 k-1} \Phi(0,0,0,0)
$$


for all $x \in \mathcal{A}$. It follows that

$$
\left\|f(x)+\frac{f(-2 x)}{2}\right\| \leq \frac{1}{2}\left[\Phi(x, x,-2 x, 0)+\frac{1}{3 k-1} \Phi(0,0,0,0)\right]
$$

for all $x \in \mathcal{A}$. Letting $u=x, v=-x$ and $w=x=0$ in (2.3), we arrive at

$$
\|f(x)+f(-x)\| \leq \Phi(x,-x, 0,0)+\frac{k+1}{3 k-1} \Phi(0,0,0,0)
$$

for all $x \in \mathcal{A}$. Making use of (2.21) and (2.22), we have

$$
\begin{aligned}
\left\|\frac{f\left(2^{l} x\right)}{2^{l}}-\frac{f\left(2^{m} x\right)}{2^{m}}\right\| & \leq \sum_{j=l}^{m-1}\left\|\frac{f\left(2^{j} x\right)}{2^{j}}-\frac{f\left(2^{j+1} x\right)}{2^{j+1}}\right\| \\
& \leq \sum_{j=l}^{m-1}\left[\left\|\frac{f\left(2^{j} x\right)}{2^{j}}+\frac{f\left(-2^{j+1} x\right)}{2^{j+1}}\right\|+\left\|\frac{f\left(-2^{j+1} x\right)}{2^{j+1}}+\frac{f\left(2^{j+1} x\right)}{2^{j+1}}\right\|\right] \\
& \leq \sum_{j=l}^{m-1} \frac{1}{2^{j+1}}\left[\Phi\left(2^{j} x, 2^{j} x,-2^{j+1} x, 0\right)+\Phi\left(2^{j+1} x,-2^{j+1} x, 0,0\right)+\frac{k+2}{3 k-1} \Phi(0,0,0,0)\right]
\end{aligned}
$$

for all integers $l, m$ with $m>l \geq 0$ and all $x \in \mathcal{A}$. So the sequence $\left\{f\left(2^{n} x\right) / 2^{n}\right\}$ is Cauchy. Since $\mathcal{M}$ is complete, the sequence $\left\{f\left(2^{n} x\right) / 2^{n}\right\}$ converges. Let $d: \mathcal{A} \rightarrow \mathcal{M}$ be a mapping defined by $(x \in \mathcal{A})$

$$
d(x):=\lim _{n \rightarrow \infty} \frac{f\left(2^{n} x\right)}{2^{n}}
$$

By letting $l=0$ and sending $m \rightarrow \infty$ in (2.9), we obtain the inequality (2.18). theorem.

The remaining part of the proof can be carried out similarly as in that of the previous

Remark 2.4. Let $f$ be a mapping from a normed algebra $\mathcal{A}$ into a Banach $\mathcal{A}$-bimodule $\mathcal{M}$. As in the previous theorems, we can prove that if $f$ satisfies the functional inequality

$$
\|f(u)+f(v)+f(w)-x f(x)-f(x) x\| \leq\left\|\frac{1}{k} f\left(k u+k v+k w-k x^{2}\right)\right\|,
$$

then $f$ is a module Jordan derivation and under suitable condition of $\Phi$, we can obtain the generalized Hyers-Ulam stability of the functional inequality

$$
\|f(u)+f(v)+f(w)-x f(x)-f(x) x\| \leq\left\|\frac{1}{k} f\left(k u+k v+k w-k x^{2}\right)\right\|+\Phi(u, v, w, x) .
$$




\section{Jacobson radical ranges of Jordan left derivations}

Every ring left derivation (resp., ring derivation) on ring is a Jordan left derivation (resp., ring Jordan derivation). The converse is in general not true. It was shown by Ashraf and Rehman [20] that a ring Jordan left derivation on a 2-torsion free prime ring is a left derivation. In particular, a famous result due to Herstein [21] states that a ring Jordan derivation on a 2torsion free semiprime ring is a derivation. In view of Thomas' result [11], derivations on Banach algebras now belong to the noncommutative setting. Among various noncommutative versions of the Singer-Wermer theorem, Brešar and Vukman [22] proved the followings: every ring left derivation on a semiprime ring is derivation which maps into its center and also every continuous linear left derivation on a Banach algebra maps into its Jacobson radical.

The followings are the functional inequality with problems as in Brešar and Vukman's result.

Theorem 3.1. Let $\mathcal{A}$ be a prime Banach algebra. Suppose that $f: \mathcal{A} \rightarrow \mathcal{A}$ is a mapping such that

$$
\|\alpha f(u)+f(v)+f(w)-2 x f(x)\| \leq\left\|\frac{1}{k} f\left(k \alpha u+k v+k w-k x^{2}\right)\right\|
$$

for all $u, v, w, x \in \mathcal{A}$ and all $\alpha \in \mathbb{U}=\{z \in \mathbb{C}:|z|=1\}$. Then $f$ is a linear left derivation which maps $\mathcal{A}$ into the intersection of its center $Z(\mathcal{A})$ and its Jacobson radical rad(A).

Proof. Let $\alpha=1 \in \mathbb{U}$ in (3.1). By Theorem 2.1, $f$ is a ring Jordan left derivation.

Setting $v=-\alpha u$ and $w=x=0$ in (3.1), we get $\alpha f(u)=f(\alpha u)$ for all $u \in \mathcal{A}$ and all $\alpha \in \mathbb{U}$. Clearly, $f(0 x)=0=0 f(x)$ for all $x \in \mathcal{A}$. Let us assume that $\lambda$ is a nonzero complex number and that $L$ a positive integer greater than $|\lambda|$. Then by applying a geometric argument, there exist $\lambda_{1}, \lambda_{2} \in \mathbb{U}$ such that $2(\lambda / L)=\lambda_{1}+\lambda_{2}$. In particular, by the additivity of $f$, we obtain $f(x / 2)=(1 / 2) f(x)$ for all $x \in \mathcal{A}$. Thus we have that

$$
\begin{aligned}
f(\lambda x) & =f\left(\frac{L}{2} \cdot 2 \cdot \frac{\lambda}{L} x\right)=L f\left(\frac{1}{2} \cdot 2 \cdot \frac{\lambda}{L} x\right) \\
& =\frac{L}{2} f\left(\left(\lambda_{1}+\lambda_{2}\right) x\right)=\frac{L}{2}\left(f\left(\lambda_{1} x\right)+f\left(\lambda_{2} x\right)\right) \\
& =\frac{L}{2}\left(\lambda_{1}+\lambda_{2}\right) f(x)=\frac{L}{2} \cdot 2 \cdot \frac{\lambda}{L} f(x)=\lambda f(x)
\end{aligned}
$$

for all $x \in \mathcal{A}$, so that $f$ is $\mathbb{C}$-linear. Therefore $f$ is a linear Jordan left derivation. Since $\mathscr{A}$ is prime, $f$ is a linear left derivation.

Note that prime Banach algebras are semiprime according to Brešar and Vukman's result which tell us that $f$ is a linear derivation which maps $\mathcal{A}$ into its center $Z(\mathcal{A})$. Since $Z(\mathcal{A})$ is a commutative Banach algebra, the Singer-Wermer conjecture tells us that $\left.f\right|_{Z(\mathcal{A})}$ maps $Z(\mathcal{A})$ into $\operatorname{rad}(Z(\mathcal{A}))=Z(\mathscr{A}) \cap \operatorname{rad}(\mathcal{A})$ and thus $f^{2}(\mathcal{A}) \subseteq \operatorname{rad}(\mathcal{A})$. Using the semiprimeness of $\operatorname{rad}(\mathcal{A})$ as well as the identity,

$$
2 f(x) y f(x)=f^{2}(x y x)-x f^{2}(y x)-f^{2}(x y) x+x f^{2}(y) x
$$

for all $x, y \in \mathcal{A}$, we have $f(\mathcal{A}) \subseteq \operatorname{rad}(\mathcal{A})$, that is, $f$ is a linear derivation which maps $\mathcal{A}$ into the intersection of its center $Z(\mathcal{A})$ and its Jacobson radical $\operatorname{rad}(\mathcal{A})$ and so the proof of the theorem is ended. 
Corollary 3.2. Let $\mathcal{A}$ be a prime Banach algebra. Suppose that $f: \mathcal{A} \rightarrow \mathcal{A}$ is a continuous mapping satisfying inequality (3.1). Then $f$ maps $\mathcal{A}$ into its Jacobson radical rad(A).

Proof. On account of Theorem 3.1, we see that $f$ is a linear left derivation on $\mathcal{A}$. Since $f$ is continuous, $f$ maps $\mathcal{A}$ into its Jacobson radical $\operatorname{rad}(\mathcal{A})$ by Brešar and Vukman's result. This completes the proof of the theorem.

With the help of the Thomas' result [11], we obtain the following.

Theorem 3.3. Let $\mathbb{A}$ be a commutative semiprime Banach algebra. Suppose that $f: \mathcal{A} \rightarrow \mathcal{A}$ is a mapping such that

$$
\|\alpha f(u)+f(v)+f(w)-x f(x)-f(x) x\| \leq\left\|\frac{1}{k} f\left(k \alpha u+k v+k w-k x^{2}\right)\right\|
$$

for all $u, v, w, x \in \mathcal{A}$ and all $\alpha \in \mathbb{U}=\{z \in \mathbb{C}:|z|=1\}$. Then $f$ maps $\mathcal{A}$ into its Jacobson radical $\operatorname{rad}(\mathcal{A})$.

Proof. Employing the same argument in the proof of Theorem 3.1, we find that $f$ is a linear Jordan derivation. Since $\mathcal{A}$ is semiprime, $f$ is a linear derivation. Thomas' result guarantees that $f$ maps $\mathbb{A}$ into its Jacobson radical $\operatorname{rad}(\mathcal{A})$, which completes the proof of the theorem.

Recall that semisimple Banach algebras are semiprime [23]. Based on that fact, the following property can be derived.

Corollary 3.4. Let $\mathcal{A}$ be a commutative semisimple Banach algebra. Suppose that $f: \mathcal{A} \rightarrow \mathcal{A}$ is a mapping satisfying inequality (3.4). Then $f$ is identically zero.

\section{Acknowledgments}

This study was financially supported by research fund of Chungnam National University in 2007. The authors would like to thank referees for their valuable comments regarding a previous version of this paper. The corresponding author dedicates this paper to his late father.

\section{References}

[1] S. M. Ulam, Problems in Modern Mathematics, chapter 6, John Wiley \& Sons, New York, NY, USA, 1964.

[2] D. H. Hyers, "On the stability of the linear functional equation," Proceedings of the National Academy of Sciences of the United States of America, vol. 27, no. 4, pp. 222-224, 1941.

[3] T. Aoki, "On the stability of the linear transformation in Banach spaces," Journal of the Mathematical Society of Japan, vol. 2, pp. 64-66, 1950.

[4] D. G. Bourgin, "Classes of transformations and bordering transformations," Bulletin of the American Mathematical Society, vol. 57, pp. 223-237, 1951.

[5] Th. M. Rassias, "On the stability of the linear mapping in Banach spaces," Proceedings of the American Mathematical Society, vol. 72, no. 2, pp. 297-300, 1978.

[6] P. Šemrl, "The functional equation of multiplicative derivation is superstable on standard operator algebras," Integral Equations and Operator Theory, vol. 18, no. 1, pp. 118-122, 1994.

[7] R. Badora, "On approximate ring homomorphisms," Journal of Mathematical Analysis and Applications, vol. 276, no. 2, pp. 589-597, 2002.

[8] D. G. Bourgin, "Approximately isometric and multiplicative transformations on continuous function rings," Duke Mathematical Journal, vol. 16, pp. 385-397, 1949. 
[9] R. Badora, "On approximate derivations," Mathematical Inequalities \& Applications, vol. 9, no. 1, pp. $167-173,2006$.

[10] I. M. Singer and J. Wermer, "Derivations on commutative normed algebras," Mathematische Annalen, vol. 129, no. 1, pp. 260-264, 1955.

[11] M. P. Thomas, "The image of a derivation is contained in the radical," Annals of Mathematics, vol. 128, no. 3, pp. 435-460, 1988.

[12] B. E. Johnson, "Continuity of derivations on commutative algebras," American Journal of Mathematics, vol. 91, pp. 1-10, 1969.

[13] O. Hatori and J. Wada, "Ring derivations on semi-simple commutative Banach algebras," Tokyo Journal of Mathematics, vol. 15, no. 1, pp. 223-229, 1992.

[14] T. Miura, G. Hirasawa, and S.-E. Takahasi, "A perturbation of ring derivations on Banach algebras," Journal of Mathematical Analysis and Applications, vol. 319, no. 2, pp. 522-530, 2006.

[15] A. Gilányi, "Eine zur Parallelogrammgleichung äquivalente Ungleichung," Aequationes Mathematicae, vol. 62, no. 3, pp. 303-309, 2001.

[16] J. Rätz, "On inequalities associated with the Jordan-von Neumann functional equation," Aequationes Mathematicae, vol. 66, no. 1-2, pp. 191-200, 2003.

[17] W. Fechner, "Stability of a functional inequality associated with the Jordan-von Neumann functional equation," Aequationes Mathematicae, vol. 71, no. 1-2, pp. 149-161, 2006.

[18] A. Gilányi, “On a problem by K. Nikodem," Mathematical Inequalities \& Applications, vol. 5, no. 4, pp. 707-710, 2002.

[19] C. Park and Th. M. Rassias, "Homomorphisms and derivations in proper JCQ*-triples," Journal of Mathematical Analysis and Applications, vol. 337, no. 2, pp. 1404-1414, 2008.

[20] M. Ashraf and N.-U. Rehman, "On Lie ideals and Jordan left derivations of prime rings," Archivum Mathematicum, vol. 36, no. 3, pp. 201-206, 2000.

[21] I. N. Herstein, "Jordan derivations of prime rings," Proceedings of the American Mathematical Society, vol. 8, no. 6, pp. 1104-1110, 1957.

[22] M. Brešar and J. Vukman, "On left derivations and related mappings," Proceedings of the American Mathematical Society, vol. 110, no. 1, pp. 7-16, 1990.

[23] F. F. Bonsall and J. Duncan, Complete Normed Algebras, vol. 80 of Ergebnisse der Mathematik und ihrer Grenzgebiete, Springer, New York, NY, USA, 1973. 